\title{
Research on Vehicle Speed Control Strategy of Multi - axis Hydrostatic Transmission
}

\author{
Liang Zhao" ${ }^{1}$ Liang Zhao ${ }^{2}$, Jin Wang ${ }^{2}$, Zhengwu Zhang ${ }^{2}$ \\ ${ }^{1}$ School of Construction Machinery, Chang’an University, Xi’an, China \\ ${ }^{2}$ School of Machinery Engineering, Shaanxi University of Technology, Hanzhong, China \\ E-mail: zhaoliangslg@163.com, wangjin63@163.com
}

Keywords: hydrostatic, driving speed, control strategy

\begin{abstract}
Aiming at the control problem of the speed of the hydrostatic transmission vehicle, the composition and working principle of the hydrostatic transmission vehicle are introduced. According to the different characteristics of the multi-axis hydraulic drive vehicle, the variable hydraulic pump control strategy is realized according to the external characteristic curve of the variable hydraulic pump and the variable hydraulic motor. The variable hydraulic motor adopts the constant Torque and constant power combined with the way of speed control strategy for multi-axis hydrostatic transmission vehicle speed control to provide a reliable theoretical basis.
\end{abstract}

\section{Introduction}

With the development of the national economy, the city's high-rise buildings more and more, with its supporting construction and fire machinery and equipment are also moving in the direction of large-scale development in order to meet the construction and fire safety requirements. In order to obtain better power and economy, we study the driving system of multi-axis hydrostatic drive chassis in terms of speed control. In order to improve the dynamic and economical performance of largescale engineering machinery and equipment with high load carrying capacity and through static hydraulic hydraulic drive Some of the theory, can solve the multi-axis hydrostatic drive chassis drive system in the theoretical lack of problems for high-speed hydraulic drive chassis speed control to provide a solution.

Large-scale machinery and equipment need to have a high carrying capacity of the chassis, and to be able to facilitate the operation, to achieve the vehicle's micro-driving and high-speed requirements. Hydrostatic transmission technology has a compact structure, wide speed range, easy to control, you can achieve the vehicle stepless speed regulation and freestyle, in the construction machinery walking system has been a lot of applications. At present, hydrostatic transmission technology is mainly used in low-speed operation of engineering vehicles, in multi-axis drive and high-speed vehicles in the application of research is still blank, speed control problems in driving lack of theoretical support.

\section{Composition and working principle}

The hydrodynamic drive vehicle has four hydraulic pumps and 14 drive motors, which are driven in a fully driven manner. Hydraulic drive vehicle unilateral driving hydraulic and control system works as shown in Fig.1, including the controller, the engine 1, the variable hydraulic pump 2, the pressure sensor 3 , the accelerator pedal 4, the handle 5, cartridge valve 6 , An electromagnetic directional valve 7 , a safety relief valve 8 , a variable hydraulic motor 9 , a speed sensor 10 , and a wheel reducer 11 . 


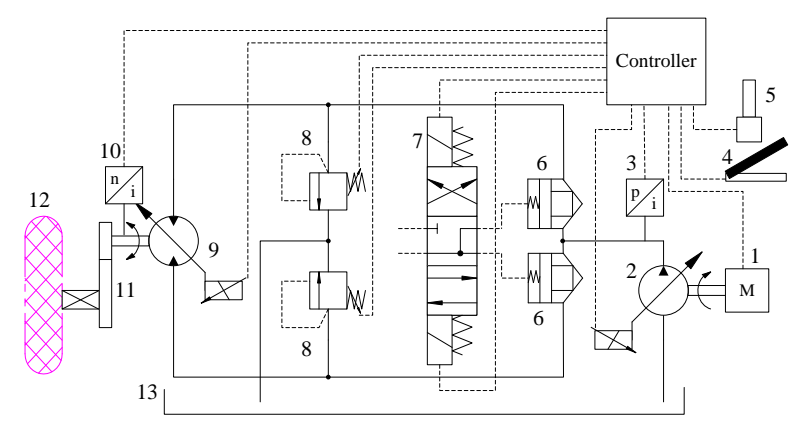

Figure 1. Schematic diagram of single-sided driving hydraulic system

The engine 1 and the variable hydraulic pump 2 are connected. The controller controls the engine 1 to drive the variable hydraulic pump by detecting the signal of the accelerator pedal. The hydraulic system is supplied to the travel system. The hydraulic motor 9 is driven by hydrostatic pressure. The wheel is driven by the wheel reducer. The electromagnetic directional control valve 7 controls the flow of the hydraulic oil to change the steering of the variable hydraulic motor and realize the function of advancing and retreating the vehicle. The hydraulic pump uses constant power control with pressure cut off, adjusts the displacement of the variable hydraulic pump according to the external characteristic curve of the engine. The variable hydraulic motor 9 is controlled by the electric proportional control. The controller detects the gear signal of the gear position and the pressure of the hydraulic system. The magnitude of the hydraulic motor coil is controlled to control the displacement of the variable hydraulic motor.

\section{Driving principle}

The control of the driving part of the multi-axis hydrostatic transmission vehicle includes the control of the engine, the variable hydraulic pump, the variable hydraulic motor and the relevant solenoid valve. The driving schematic diagram shown in Fig.2. The engine speed is adjusted by adjusting the throttle opening of the engine to adjust the throttle opening of the engine. The maximum power of the engine at each speed is determined according to the external characteristic curve of the engine, and the target displacement of the variable hydraulic pump is determined according to the pressure of the hydraulic system. Through the stall handle stall signal and hydraulic system pressure adjustment variable hydraulic motor displacement, change the speed of the variable hydraulic motor to change the speed of the vehicle.

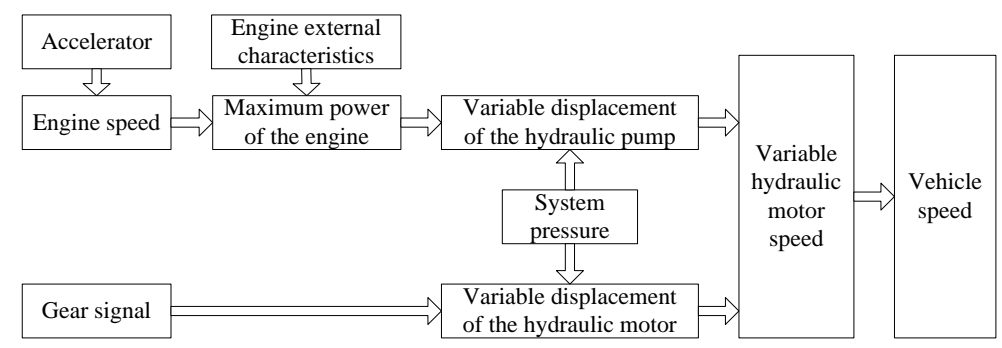

Figure 2. Schematic diagram of the operation of the hydrostatic transmission vehicle

\section{Division of travel vehicles}

Multi-axis hydrostatic transmission vehicles in the transmission system without gearbox, the gear design is based on the number of drive axles to determine the number. When the vehicle is moving forward, the number of axles is changed from 7 to 3, and the number of axles is divided into five gears according to the number of drive axles. Can be manual and automatic gear switch, when the need to drive the number of less when the corresponding turn off the displacement of the hydraulic motor, let it in a zero displacement state, to improve the speed of other drive hydraulic motor. 


\section{Hydraulic pump control strategy}

\subsection{Hydraulic Pump Performance}

Rexroth's A11VO190 hydraulic variable pump is selected according to the requirements of the hydraulic flow rate of the vehicle. The electrical control of the proportional electromagnets allows the displacement of the pump to be stepless and programmable. The displacement of the pump is proportional to the electromagnetic force (current strength). The control force on the control piston is generated by the proportional solenoid. In order to control the proportional solenoid, 24V (EP2) DC power supply. Control direction from Vgmin-Vgmax, control current increases, pump displacement increases. Control start point: $200 \mathrm{~mA}$ (EP2) Control end point: $600 \mathrm{~mA}$ (EP2), the performance curve as shown:

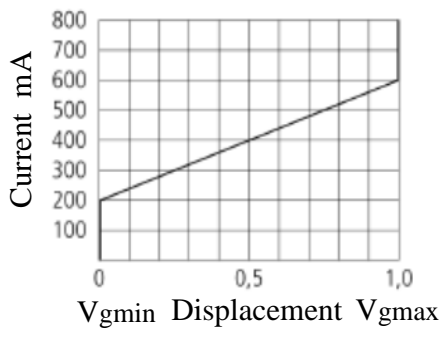

Figure 3. variable hydraulic pump performance curve

\subsection{Hydraulic Pump Control Strategy}

The hydraulic pump is directly connected to the engine, the input speed, torque and engine output speed, torque and power of the hydraulic pump are equal.

The external characteristics of the engine are shown in Fig.4, where $n_{1}$ is the idle speed of the engine; $n_{2}$ is the engine maximum output torque when the speed; $n_{3}$ is the engine maximum output torque when the speed.

Considering the economy of the engine, the maximum displacement control of the hydraulic pump at each speed is:

$$
q_{b}=\frac{0.9 M_{e} 2 \pi}{\Delta p}
$$

Where $q_{b}$ is the displacement of the hydraulic pump; $\Delta p$ is the pressure difference between the outlet and inlet of the hydraulic pump.The displacement control curve of the hydraulic pump is controlled along the curve $\mathbf{1}^{\prime}-\mathbf{2}-\mathbf{3}$ in Figure5.

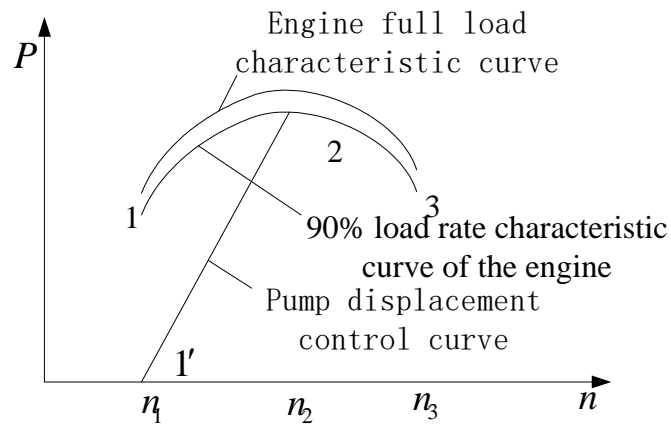

Figure 4. Maximum displacement control curve for hydraulic pumps

According to the external characteristics of the engine to control the displacement of the hydraulic pump, when the engine load rate of $90 \%$, the engine fuel consumption is the best. The displacement control of the hydraulic pump is as follows:

$n_{0} \leq n \leq n_{M \max }$ : 


$$
q_{b}=60000 \times \frac{0.9 P_{M \max }\left(n-n_{0}\right)}{n_{M \max }-n_{0}} \times \frac{\eta}{P_{p} \cdot n}
$$

$n_{M \max } \leq n:$

$$
q_{b}=60000 \times 0.9 \times P_{M \max } \times \frac{\eta}{P_{P_{\max }} \cdot n}
$$

Where $q_{b}$ is the displacement of the hydraulic pump; $n$ is the speed of the engine; $P_{M \max }$ is the power that the pump can supply when the engine is at maximum torque; $n_{M \text { max }}$ is the engine speed when the maximum torque; $n_{\mathbf{0}}$ is the speed at which the pump displacement starts; $\eta$ is efficiency; $P_{P}$ is the system pressure; $P_{M \max }$ is the system maximum pressure.

Hydraulic pump control strategy shown in Fig. 5.

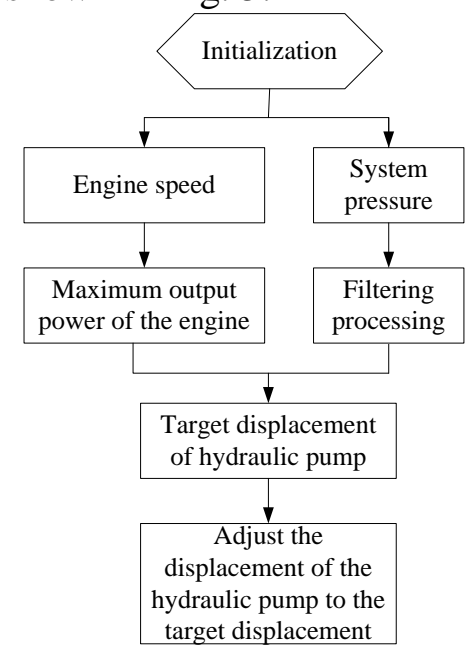

Figure 5. Hydraulic pump control strategy

\section{Hydraulic motor control strategy}

\subsection{Hydraulic Motor Performance}

According to the vehicle's driving conditions, in addition to meet the requirements of speed, but also to be able to zero displacement, the choice of Rexroth's A6VM200 variable hydraulic motor. Electric control belt proportional solenoid can be controlled according to the electrical signal stepless control motor displacement, the control function is proportional to the applied current, the variable hydraulic motor control voltage is $24 \mathrm{~V}$, the control starting current is $200 \mathrm{~mA}$ control the end current is $600 \mathrm{~mA}$, the hydraulic motor Displacement control is anti-proportional control at a current of $600 \mathrm{~mA}$ when the hydraulic motor displacement is zero, the performance curve as shown in Fig. 6:

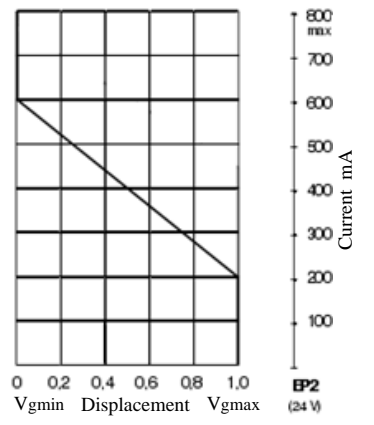

Figure 6 .Variable hydraulic motor performance curve 


\subsection{Hydraulic Motor Control Strategy}

First, the motor displacement opening to the maximum, and then according to the highest speed of each file to determine the motor displacement, the motor displacement from the maximum transfer to the maximum speed corresponding to the displacement, and then automatically adjust the motor displacement according to the system pressure.

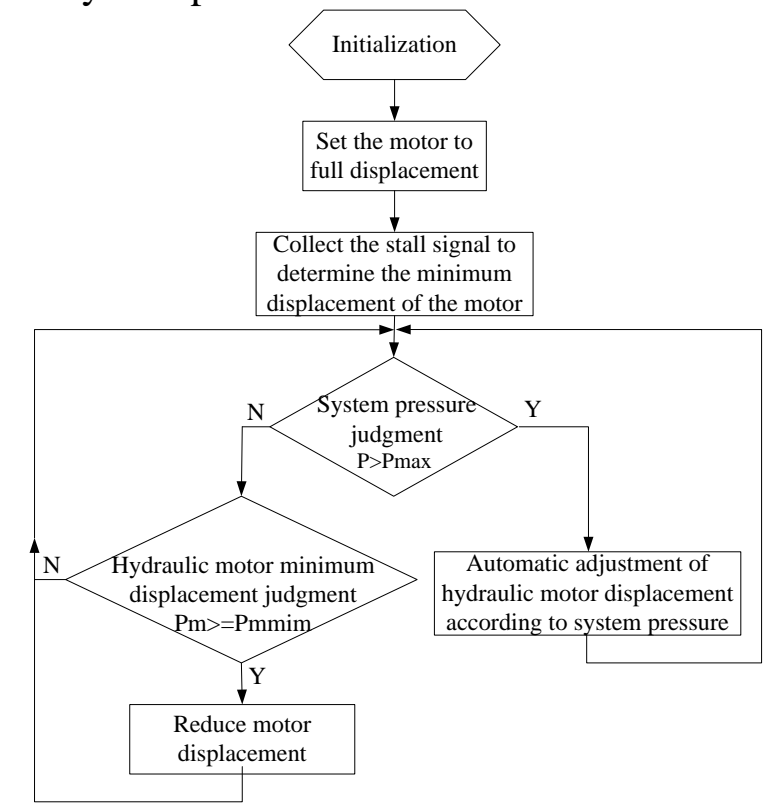

Figure 7. Control strategy for hydraulic motors

The motor displacement in accordance with the speed changes to adjust at any time, speed and force into a similar hyperbolic relationship, resulting in constant power control, according to the current speed calculated motor target displacement:

$$
q_{m}=\frac{q_{\min } \cdot v_{\max }}{v}
$$

Where $q_{m}$ is the displacement of the hydraulic motor; $\quad v$ is the speed of the vehicle; $\quad v_{\max }$ is the maximum speed per file; $q_{\min }$ is the minimum displacement corresponding to each gear of the motor.

\section{Conclusion}

Based on the working principle of hydraulic hydraulic drive vehicle, the control strategy of variable hydraulic pump and variable hydraulic motor is put forward according to the performance of hydraulic pump and hydraulic motor.

(1) According to the engine's external characteristics of the variable displacement of the hydraulic pump control, the engine fuel consumption is the best;

(2) According to the driving conditions of the vehicle to control the displacement of the variable hydraulic motor, the torque required at the time of starting is large, and the variable hydraulic control of the variable hydraulic motor is used to maximize the displacement of the hydraulic motor. Constant power control, regulation of hydraulic motor displacement;

(3) The study of speed control strategy provides the theoretical basis for the design of hydrostatic transmission vehicle.

\section{Acknowledgment}

The article was supported by Special research project of Shaanxi Provincial Department of Education (Grant No. 14JK1134), Shaanxi Province Natural Science Basic Research Project (Grant 
No. 2017JM5087).

\section{References}

[1] Liu Peng, Zhang Runli, Liu Xinfu. "Research on Matching Method of Hydrostatic Transmission Engine and Variable Pump Power”. Engineering Machinery Abstracts, 2012(4),pp.43-45

[2] Chen Jinquan, Hu Junke, Zhang Zhengming. "Application of Hydrostatic Driving System in High - speed Off - road Forklift”. Modern manufacturing engineering, 2010(4),pp.123-127.

[3] Liang Xiaodong, Feng Maolin, Sun Fen. "Research on Double Pump Combined Technology of Hydraulic System”. Colored equipment, 2011(1),pp.14-16.

[4] M Comellas J, Pijuan X Potau . "Analysis of a hydrostatic transmission driveline for its use in off-road multiple axle vehicles”. Journal of Terramechanics, 2012, (5),pp. 245-254.

[5] IZQUIERDO J, IGLESIAS P L. "Mathematical Modelling of Hydraulic Transients in Simple Systems”.Mathematical and Computer Modelling, 2002, (7),pp.801-812.

[6] Mohamed Saber Ahmed Ibrahim-Sokar.Verlag.Investigation of Hydraulic Transmissions for Passenger Cars. Diss. RWTH Aachen University,2011

[7] ZHAO Hui, JIA Xiaoping. "The propulsion system designing and researching of $6 \times 6$ wholehydraulic vehicle”. Machinery Design \& Manufacture, 2007,(4),pp.26-27.

[8] YI Xiaogang, WANG Xin. “Matching and control techniques for hydrostatic grader”.Road Machinery \& Construction Mechanization, 2008, (3),pp.18-21.

[9] TIAN Jinyue,YU Ying. "Research on performance of hydrostatic transmissions of vehicles”. Transactions of the Chinese Society for Agricultural Machinery, 2002, (4),pp.32-34.

[10] ZHAO Liang. "Matching and control study on driving system of full hydraulically-driven chassis”,Journal of China Construction Machinery Corporation, 2013,(6),pp.490-493

[11] J. Clerk Maxwell, A Treatise on Electricity and Magnetism, 3rd ed., vol. 2. Oxford: Clarendon, 1892, pp.68-73. 